\title{
Essais
}

Revue interdisciplinaire d'Humanités

Narration et lien social

\section{Négocier la Censure}

À propos d'Edoardo Tortarolo, L'invenzione della libertà di stampa.

Censura e scrittori nel Settecento, Rome, Carocci, 2011

Sandro Landi

\section{OpenEdition}

1 Journals

Édition électronique

URL : https://journals.openedition.org/essais/9559

DOI : 10.4000 /essais.9559

ISSN : 2276-0970

Éditeur

École doctorale Montaigne Humanités

Édition imprimée

Date de publication : 15 mai 2013

Pagination : 207-212

ISBN : 978-2-9544269-0-7

ISSN : 2417-4211

Référence électronique

Sandro Landi, « Négocier la Censure », Essais [En ligne], 3 | 2013, mis en ligne le 01 septembre 2021, consulté le 08 septembre 2021. URL : http://journals.openedition.org/essais/9559; DOI : https:// doi.org/10.4000/essais.9559 


\title{
Négocier la Censure À propos d'Edoardo Tortarolo, L'invenzione della libertà di stampa. Censura e scrittori nel Settecento, Rome, Carocci, 2011
}

\author{
Sandro Landi
}

La publication de ce tout nouveau livre sur l'histoire de la censure offre indirectement l'opportunité de réfléchir sur la nature et les limites de la liberté d'expressions dans notre société : sans doute est là l'une des raisons qui expliquent la multiplication des études et des synthèses à ce sujet. Cette demande d'histoire semble révéler un certain malaise vis-à-vis de la censure, un phénomène que l'historiographie a longtemps classé parmi les images d'un passé révolu et familier, mais que le présent nous oblige soudain à repenser dans son actualité. Face aux transformations qui affectent aujourd'hui l'accès de tout discours à la sphère publique, notre perception de la censure est en train de changer ainsi que les catégories interprétatives utilisées dans son approche historique. Le dernier ouvrage d'Edoardo Tortarolo, consacré aux modèles de censure et aux débats sur la liberté de la presse dans l'Europe du XVIII siècle, témoigne de ce changement dont il convient de rappeler brièvement les enjeux.

Tout d'abord, l'histoire de la censure s'est progressivement émancipée d'une représentation de la réalité de type dualiste. Selon cette représentation, dont les origines protestantes remontent au $\mathrm{XVI}^{\mathrm{e}}$ siècle $^{1}$, le livre imprimé est, en soi, vecteur de liberté individuelle et de progrès civil. Dans cette lecture, l'histoire de la censure est, à proprement parler, l'histoire d'une négation qui se résume en une lutte en vue de son abrogation; l'article 11 de la Déclaration de l'homme et du citoyen (1789), qui inscrit la liberté de la presse au nombre des droits inaliénables de l'individu, constitue son aboutissement inévitable. Cette interprétation, longtemps dominante dans les synthèses sur histoire du livre et de la lecture est aujourd'hui soumise à une révision substantielle. Plusieurs facteurs ont contribué à rendre cette image obsolète : d'une part le renouvellement des études sur la censure ecclésiastique suite en particulier à la réouverture au public des archives des congrégations romaines du Saint-Office et de l'Index.

1 A. Johns, The Nature of the Book. Print and Knowledge in the Making, Chicago-London, 1988, University of Chicago Press. 
L'extraordinaire richesse de cette documentation a permis une connaissance plus approfondie de la censure ecclésiastique, modèle et archétype des autres censures, tout en contribuant à réduire le risque d'anachronisme qui pèse sur son interprétation ${ }^{2}$. La censure ecclésiastique ou laïque à l'époque moderne n'est ni un organisme monolithique, ni un dispositif cohérent et efficace de suppression des idées - comparable à celui mis en place par les États totalitaires du XXe siècle -, mais plutôt un ensemble de pratiques institutionnelles et culturelles qui, trois siècles durant, ont déterminé les conditions d'existence publique de tout discours imprimé.

D’autre part, la censure étant envisagée comme un phénomène extra-institutionnel participant aux processus de communication des sociétés modernes, apparaît comme le dispositif langagier qui rend possible, c'est-à-dire légitime, l'existence publique d'un discours. Les sources théoriques de cette banalisation de la notion de censure sont multiples, mais la réception historiographique des thèses de Pierre Bourdieu et de Léo Strauss a été, à cet égard, décisive ${ }^{3}$. Bourdieu comme Strauss ont en effet mis en évidence la dimension historique de l'autocensure, conçue pour le premier, comme un acte implicite, inhérent au processus de création et de légitimation du discours, alors que pour le second, il s'agit d'une stratégie de communication oblique, visant à repérer un noyau de lecteurs capables de «lire entre les lignes".

Dernier grand élève du maitre turinois Franco Venturi, spécialiste réputé de la culture politique européenne au XVIII ${ }^{e}$ siècle, Edoardo Tortarolo propose une interprétation de la censure qui échappe au double écueil de la téléologie et du révisionnisme. Dans son ouvrage, la censure garde l'épaisseur d'un phénomène institutionnel, encore profondément ancré, dans le XVIII siècle, dans les processus de publication et dans les modes de fonctionnement des sociabilités littéraires. Le premier mérite incontestable de ce livre consiste donc à contourner le lieu commun historiographique qui a tendance à juxtaposer les " lumières " à la censure. Plus précisément, Tortarolo étudie cette phase du développement de la censure à partir des connaissances et des questionnements développés par les historiens de la première époque moderne. Vue sous cet angle, qui prend en compte une temporalité longue, la liberté de la presse perd son caractère absolu et apparaît comme une possibilité latente des systèmes de censure : une possibilité qui consiste à produire des espaces semi-institutionnels, non rigides et toujours négociés de liberté d'expression.

Le périmètre de cette recherche est très large tant sur le plan temporel que spatial. Après une introduction consacrée à la censure comme enjeu historiographique, Tortarolo examine les propositions philosophiques de Spinoza

2 A. Prosperi, L'Inquisizione romana. Letture e ricerche, Rome, Edizioni di Storia e Letteratura, 2003.

3 P. Bourdieu, «La censure », in Questions de sociologie, Paris, Les Éditions de Minuit, p. 138-142;

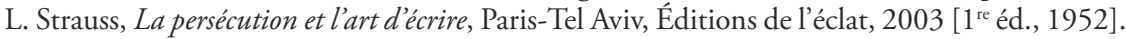


(Tractatus theologico-politicus) et de Hobbes (Leviathan) qui sont, au niveau européen, à l'origine de deux modèles opposés de concevoir la liberté d'expression. Il s'intéresse ensuite à l'Angleterre et à la période qui suit l'abolition tacite de la censure préventive (non reconduction du Licensing act, 1695). Puis il consacre deux chapitres à la censure dans le royaume de France (le premier traite des positions des philosophes vis-à-vis de la liberté de la presse et le second de l'évolution de la censure préventive jusqu'à son abolition à la veille de la Révolution). Il termine par une analyse de quelques variantes continentales de la censure (États italiens, Empire des Habsbourg, Prusse, Danemark, Hollande).

Essayons maintenant de préciser le principal résultat qui émerge de cette importante enquête. Nous remarquons que dans tous les cas étudiés, la censure n'est pas au XVIII ${ }^{\mathrm{e}}$ siècle une réalité résiduelle, mais un dispositif complexe, capable d'intégrer, dans certaines limites, les sollicitations et les critiques de la société civile et de rester fonctionnel jusqu'à la fin du siècle. La clé pour comprendre la longévité de ces systèmes se résume à deux formules : «liberté négociée » et " ambiguïté fonctionnelle ». La censure est en effet un dispositif dynamique, capable de délimiter l'espace du dicible tout en s'ouvrant à de nouvelles propositions intellectuelles. L'exemple le plus éclatant d'ambigüité fonctionnelle est celui des "permissions tacites ", c'est-à-dire de la licence d'imprimer accordée confidentiellement par certaines censures d'état à des ouvrages qui remplissent les critères nécessaires pour une publication tolérée mais non autorisée. Il s'agit d'une pratique institutionnalisée notamment en France et dans d'autres pays européens et qui implique, comme le montre bien Tortarolo, une coopération entre auteurs, censeurs et éditeurs dans le processus de publication d'un ouvrage. Les acteurs qui interviennent dans ce circuit de publication aux marges de la légalité - mais largement admis et encouragé - sont par ailleurs souvent interchangeables. Le modèle de censure en vigueur dans les principaux états européens d'ancien régime est donc un système qui rend possible une conception de la liberté de la presse paradoxale mais largement partagée et acceptée par la grande majorité des acteurs qui interviennent dans le processus de publication.

Cependant, malgré son incontestable fonctionnalité, ce modèle s'écroule soudain à la fin du XVIII ${ }^{e}$ siècle. Pourquoi, se demande Tortarolo, ce système institutionnel, différemment articulé dans les différents pays mais fondé sur une extrême responsabilisation de tous les participants à la production du livre, a-t-il perdu rapidement toute crédibilité sans être remplacé, après son abolition, par rien de comparable $?^{4}$

C'est une question qui reste en partie ouverte et qui justifie quelques remarques. La première concerne la physionomie des systèmes de censure décrite dans cet ouvrage. E. Tortarolo étudie l'évolution de la censure préventive.

4 E. Tortarolo, Linvenzione della libertà di stampa. Censura e scrittori nel Settecento, Rome, Carocci, 2011, p. 160. 
Celle-ci présente une typologie assez riche dans les différents cas étudiés, mais elle comporte partout l'existence d'un rituel qui, par son caractère complexe et désuet, a attiré jusqu'à présent l'attention presque exclusive des historiens. Toutefois, à côté d'une censure préventive existe partout une censure postérieure à l'impression et qui constitue la forme la plus ordinaire et durable de contrôle de l'industrie typographique. En effet, prévention et répression constituent souvent deux aspects concomitants du même phénomène. Toutefois, si la prévention est inconcevable sans la répression, la censure répressive, en revanche, peut facilement subsister de façon autonome. C'est ainsi, par exemple, que l'abolition tacite de la censure préventive donne lieu en Angleterre à une répression accrue des libelles considérés comme séditieux et blasphématoires ${ }^{5}$ et qu'en France, malgré la Déclaration de 1789, qui inaugure officiellement l'ère de la liberté de la presse, la chasse aux écrits antirévolutionnaires est féroce sous la Terreur ${ }^{6}$. Il est vrai que la censure préalable n'est pas rétablie sous l'Empire, mais la censure napoléonienne, redoutablement efficace, soumise directement aux ordres du ministère de la police, constitue un véritable modèle pour les États européens à l'époque de la Restauration 7 . Si l'on suit, dans les différents cas européens la dynamique censure préventive/censure répressive, force est alors de constater que la critique et l'effondrement de la première mène partout à une consolidation de l'appareil de contrôle policier des imprimés. À la lumière de ce processus de réforme et de réoccupation fonctionnelle de la censure, la crise de ces systèmes n'est pas simplement une question d'intellectuels mais aussi et surtout de fonctionnaires. En d'autres termes, parmi les causes de la disparition soudaine de la censure préventive, la critique interne, institutionnelle de ce dispositif, formulée par des censeurs et des bureaucrates, joue un rôle essentiel.

Je me limite à un exemple significatif. En 1771, le ministre des affaires étrangères de la cour de Vienne, Von Kaunitz, se déclare favorable à un régime de « liberté de la presse » concernant l'État de Milan ${ }^{8}$. Cependant, précise-t-il, le but de cette liberté, conçue comme " apparente ", consiste principalement à invalider la censure préventive, confiée à des intellectuels trop enclins à la négociation et au compromis avec d'autres intellectuels. Que laisse entendre le discours de Kaunitz? Tout d'abord que la liberté de la presse, dans la seconde

5 P. McDowell, The Women of Grub Street: Press, Politics, and Gender in the London Literary Marketplace, 1678-1730, Oxford, Clarendon Press, 1988; Ph. Hamburger, "The Development of the Law of Seditious Libel and the Control of the Press", in Stanford Law Review, n ${ }^{\circ} 37$, 1984, p. 661-765.

6 C. Walton, Policing Public Opinion in the French Revolution: The Culture of Calumny and the Problem of Free Speech, Oxford, Oxford University Press, 2009.

7 B. Vouillot, "La Révolution et l'Empire : une nouvelle réglementation ", in Histoire de l'édition française, II, Le livre triomphant (1660-1830), sous la direction de R. Chartier et H.-J. Martin, Paris, Fayard, 1989, p. 694-708.

8 A. Tarchetti, "Censura e censori di sua maestà imperiale nella Lombardia austriaca: 1740-1780", in Economia, istituzioni, cultura in Lombardia nell'età di Maria Teresa, III, Bologne, Il Mulino, 1984, p. 741-792. 
moitié du XVIII siècle, est désormais devenue un lieu commun et presque un slogan du discours politique et journalistique. Tout gouvernement se doit de s'approprier cette formule dans le but de promouvoir son image auprès de cette instance impersonnelle et supranationale que l'on qualifie déjà à cette époque d'«opinion publique ». C'est ainsi à la lumière des tensions et des dynamiques propres à chaque administration de la censure, que nous pouvons saisir les enjeux contenus dans une expression telle que "liberté de la presse » qui n'est en réalité qu'un véritable trompe-l'œil. Cette expression nous oblige à distinguer entre une réalité verbale, c'est-à-dire entre un vocabulaire normatif dont elle fait partie par effet du lexique dominant des Lumières, et les pratiques de limitation de la liberté d'expression concrètement à l'œuvre dans chaque État. Ensuite, le discours du haut-fonctionnaire impérial nous indique que la critique et l'abolition, de facto ou officielle, de la censure préventive, loin de déboucher sur un régime de liberté effective d'expression, mène plutôt à une judiciarisation de la censure : celle-ci, dès la fin du XVIII siècle, échappe en effet aux intellectuels et devient l'un des domaines spécifiques des systèmes de police?.

Une deuxième remarque porte sur l'existence même d'un modèle de censure continentale, fondé selon E. Tortarolo, sur la notion de " liberté négociée ». Il ne fait aucun doute que les censures européennes - grâce aussi à une circulation de modèles et d'expériences - présentent des traits comparables. Cependant, l'uniformisation de certaines pratiques, comme celle des permissions tacites, ne doit pas empêcher de souligner des spécificités parfois irréductibles. Celle qui concerne le rapport entre censure et opinion publique qui s'établit dans les différents contextes politiques d'ancien régime en est une. À partir des années 1770, dans le discours politique des Lumières, l'« opinion publique » est conçue comme un "tribunal " impersonnel et impartial susceptible de remplacer la censure absolutiste dans le rôle de filtre de tout ouvrage imprimé ${ }^{10}$. À la même époque, dans les États germaniques mais aussi dans les États italiens gouvernés par la maison de Habsbourg, l'opinion publique n'est pas conçue comme une instance critique, mais plutôt comme une sphère de discussion sur des sujets d'intérêt public, une sphère solidaire avec les orientations générales du gouvernement. C'est une physionomie de l'espace public qui a été soulignée par bon nombre d'études récentes - dont celles, fondamentales, d'E. Tortarolo ${ }^{11}-$ qui ont le mérite de rendre le modèle habermassien d'opinion publique - conçu sur la base d'une généralisation de l'expérience anglaise et française - inopérant dans la plupart des cas européens ${ }^{12}$.

9 Cf. S. Landi, Stampa, censura, opinione pubblica in età moderna, Bologne, Il Mulino, 2011, p. $87-92$.

10 Tortarolo, Linvenzione della libertà di stampa, op. cit., p. 162.

11 E. Tortarolo, La ragione interpretata. La mediazione culturale tra l'Italia e la Germania nell'età dell'Illuminismo, Rome, Carocci, 2003.

12 Cf. S. Landi, «Au-delà de l'espace public. Habermas, Locke et le consentement tacite ", in Revue d'histoire moderne et contemporaine, 59-4, 2012, p. 7-31. 
En effet, penser l'opinion publique comme un acteur nécessairement autonome, rationnel et critique à l'égard du pouvoir politique est une pure abstraction. En revanche, penser la censure non pas comme un résidu archaïque, mais comme un élément constitutif de la sphère publique moderne permet de comprendre sa surprenante actualité. Le livre d'E. Tortarolo nous offre à ce propos des clés de lecture indispensables.

Sandro Landi

Professeur des universités

EA $4574 \mathrm{SPH}$

Université Michel de Montaigne Bordeaux 3 Sandro.Landi@u-bordeaux3.fr 